\title{
Swampland bounds on the Abelian gauge sector
}

\author{
Seung-Joo Lee ${ }^{1, *}$ and Timo Weigand ${ }^{1,2, \uparrow}$ \\ ${ }^{1}$ CERN Theory Department, 1 Esplanade des Particules, CH-1211 Geneva, Switzerland \\ ${ }^{2}$ PRISMA Cluster of Excellence and Mainz Institute for Theoretical Physics, Johannes Gutenberg-Universität, \\ 55099 Mainz, Germany
}

(Received 8 June 2019; published 24 July 2019)

\begin{abstract}
We derive bounds on the number of Abelian gauge group factors in six-dimensional gravitational theories with minimal supersymmetry and in their $F$-theoretic realizations. These bounds follow by requiring consistency of certain Bogomol'nyi-Prasad-Sommerfield strings in the spectrum of the theory, as recently proposed in the literature. Under certain assumptions, this approach constrains the number of Abelian gauge group factors in six-dimensional supergravity theories with at least one tensor multiplet to be $N \leq 20$ (or $N \leq 22$ in the absence of charged matter). For any geometric $F$-theory realization with at least one tensor multiplet, we establish the bound $N \leq 16$ by demanding unitarity of a heterotic solitonic string which exists even in the absence of a perturbative heterotic dual. This result extends to four-dimensional $F$-theory vacua on any blowup of a rational fibration. Our findings lead to universal bounds on the rank of the Mordell-Weil group of elliptically fibered Calabi-Yau 3-folds.
\end{abstract}

DOI: 10.1103/PhysRevD.100.026015

\section{INTRODUCTION}

It is widely believed that the compatibility of a gauge theory with quantum gravity imposes extra constraints on the structure of the theory, which may not be visible from the consistency of the low-energy effective theory alone. The resulting schism of theory space into a swampland [1] of theories lacking an embedding into quantum gravity versus the landscape of fully consistent theories including gravity is a subject that has attracted considerable recent attention $[2,3]$.

In this paper, we report on new bounds on the maximal number of Abelian gauge group factors in six-dimensional gravitational theories with $\mathcal{N}=(1,0)$ supersymmetry. This class of theories is known to be highly constrained by the consistent cancellation of gauge and gravitational anomalies. Local anomaly considerations have lead to a number of remarkable restrictions on the architecture of the gauge sector of six-dimensional supergravities, such as those found in Refs. [4-6] (see Ref. [7] for background and more references). Apart from being interesting in itself, this line of reasoning can also be viewed as a first step towards studying similar questions in a four-dimensional context.

\footnotetext{
*seung.joo.lee@cern.ch

timo.weigand@cern.ch
}

Published by the American Physical Society under the terms of the Creative Commons Attribution 4.0 International license. Further distribution of this work must maintain attribution to the author(s) and the published article's title, journal citation, and DOI. Funded by SCOAP ${ }^{3}$.
Abelian gauge symmetries play a distinguished role in the swampland program: unlike their non-Abelian counterparts, they must necessarily couple to gravity [8] in order for their anomalies to be canceled by a Green-Schwarz mechanism. It is even more surprising that anomalies alone do not seem to give a universal bound on the number of Abelian gauge group factors even in the highly constrained setup of six-dimensional supergravities with eight supercharges. In Ref. [5] it was observed that the number of Abelian gauge group factors can in principle be infinite, as far as the structure of gauge-gravitational anomalies is concerned, at least in the absence of charged hypermultiplet matter and as long as the theory contains more than eight tensor multiplets in its spectrum. For $T \leq 8$ tensor multiplets, on the other hand, Ref. [5] determined the bound on the number of Abelian gauge group factors in purely Abelian theories to be [9]

$$
N \leq(T+2)\left(T+\frac{7}{2}+\left(T^{2}-51 T+\frac{2225}{4}\right)^{1 / 2}\right),
$$

which gives the upper bounds

$$
\{54,81,107,134,160,185,211,236,260\},
$$

for $T=0,1, \ldots, 8$, respectively, while for $T=0$ the stronger bound $N \leq 17$ has been found.

As pointed out very recently in Ref. [10], extra constraints have to be imposed on a six-dimensional (6D) supergravity theory by demanding unitarity of the sector of Bogomol'nyi-Prasad-Sommerfield (BPS) strings coupling 
to the tensor multiplets. In this paper we observe that the resulting consistency conditions severely constrain the maximal number of possible Abelian gauge group factors, whether or not a non-Abelian gauge sector is present. In a pure supergravity analysis, bounds can be established modulo certain assumptions on the integrality and generic nondegeneracy of a class of BPS strings. With these assumptions, in Sec. III, we will bound the number of Abelian gauge group factors to be $N \leq 20$ for $T \geq 1$ in the presence of charged matter [and $N \leq 22$ if some $U(1)$ 's have no charged matter] and $N \leq 32$ for $T=0$. This rules out the possibility of an infinite number of Abelian gauge group factors, provides a definite bound for $T=0$ even in the presence of a non-Abelian gauge sector, and considerably improves the above-mentioned bound (1) for $1 \leq T \leq 8$.

While the supergravity analysis relies on certain assumptions on the string charge spectrum, the situation is even clearer once we turn to explicit realizations of 6D $\mathcal{N}=(1,0)$ supergravities in $F$-theory in Sec. IV. For all such $F$-theory models with $T \geq 1$ we are able to constrain the maximal number of Abelian group factors to be

$$
N \leq 16
$$

by considering a heterotic string obtained by wrapping a D3-brane on a distinguished curve on the $F$-theory compactification space. The appearance of this heterotic string, which exists even in the absence of a perturbative heterotic dual, has played a crucial role in the study of swampland and weak gravity conjectures in Refs. [11-14]. Our constraint (3) heavily relies on the results of Ref. [8] concerning the form of the so-called height pairing of rational sections describing Abelian gauge groups in $F$-theory.

Our main message is that Abelian gauge groups in sixdimensional $F$-theory models with $T \geq 1$ can always be embedded in the $E_{8} \times E_{8}$ current algebra on the abovementioned heterotic string, even if no perturbative heterotic dual exists. This is a notable difference to non-Abelian gauge groups. Combined with the weaker bound $N \leq 32$, which we will derive for $F$-theory models with $T=0$, this predicts a universal bound on the number of independent nontorsional rational sections on any elliptically fibered Calabi-Yau 3-fold. To date no such bound has been derived in the mathematics literature.

In Sec. V we speculate on extensions of our results to $F$ theory compactifications to four dimensions: as long as the base of the elliptic fibration is the blowup of a rational fibration, we expect the bound $N \leq 16$ to continue to hold for all Abelian gauge group factors which are associated with rational sections. We furthermore conjecture that the bound $N \leq 16$ should also hold for six-dimensional $F$-theory models with $T=0$, which would even improve the existing conditional bound $N \leq 17$ obtained from anomaly cancellation in the absence of non-Abelian gauge dynamics. Finally, our results on six-dimensional $F$-theory with $T \geq 1$ should also constrain the charge pattern for Abelian gauge groups, as these must be embeddable into $E_{8} \times E_{8}$.

\section{BACKGROUND AND REVIEW}

Consider a $6 \mathrm{D} \mathcal{N}=(1,0)$ supergravity theory with vector multiplets in the gauge group $G=\prod_{l} G_{l}$, where $G_{l}$ are simple non-Abelian factors, as well as $T$ tensor multiplets. Each tensor multiplet contains one antichiral tensor $B_{\alpha}^{-}, \alpha=1, \ldots, T$, along with a real scalar. In addition, the gravity multiplet contains a self-dual tensor $B^{+}$. The structure of gauge and gravitational anomalies of this theory is characterized by the anomaly coefficients $b_{G_{t}}$ and $a$, which can be represented as vectors in $\mathbb{R}^{1, T}$, endowed with an intersection product of signature $(1, T)$. The gravitational anomaly coefficient $a$ must satisfy the relation $a \cdot a=9-T$. This is solved, for instance, by the vector $-a=(3,-1, \ldots,-1) \in \mathbb{R}^{1, T}$, where we choose the inner product to be $\eta_{A B}=\operatorname{diag}(1,-1, \ldots,-1$ ) (for $A \in\{0, \alpha\})$. The real scalars in the tensor multiplet are assembled in a vector $j \in \mathbb{R}^{1, T}$ subject to the normalization condition $j \cdot j=1$. The inverse gauge coupling squared of the vector multiplets is controlled by the scalars in the tensor multiplet as

$$
\frac{1}{g_{G_{l}}^{2}}=j \cdot b_{G_{l}}
$$

The tensor fields couple to BPS strings whose worldsheet theory is described by a two-dimensional (2D) $\mathcal{N}=(0,4)$ theory. From the perspective of the supergravity theory alone the BPS strings are characterized by an integral charge vector $Q$ in a unimodular lattice [15] of tension $j \cdot Q$. As in Ref. [10], we are interested in strings that do not correspond to instanton strings in 6D $\mathcal{N}=(1,0)$ superconformal field theories (SCFTs) once gravity is decoupled or in little string theories (LSTs). An analysis of the anomaly polynomial of these strings reveals that the left- and right-moving central charges of the 2D SCFTs to which the worldsheet theories flow in the infrared (IR) are given by (see Ref. [10] and references therein)

$c_{L}=3 Q \cdot Q-9 Q \cdot a+2, \quad c_{R}=3 Q \cdot Q-3 Q \cdot a$.

In these expressions, the contribution from the free hypermultiplet describing the center-of-mass motion of the string has already been removed. Furthermore, Eq. (5) relies on the identification of the $R$ symmetry of the $2 \mathrm{D}$ worldsheet SCFT in the IR with the factor $S U(2)_{R}$ in the decomposition $S O(4)=S U(2)_{R} \times S U(2)_{l}$, where $S O(4)$ is the rotation group acting on the extended directions transverse to the string. This is the correct identification for the strings that are not associated with 6D SCFTs or LSTs [10]. 
The gauge group $G$ of the 6D supergravity as well as the $S U(2)_{l}$ symmetry act as flavor symmetries on the string, with 't Hooft anomaly coefficients

$$
k_{G_{l}}=Q \cdot b_{G_{i}}, \quad k_{l}=\frac{1}{2}(Q \cdot Q+Q \cdot a+2) .
$$

In the IR 2D SCFT, these take the role of the Kac-Moody level of the worldsheet current realizing the respective flavor symmetry (see, e.g., Ref. [16] for background). The unitarity of the worldsheet SCFT demands that $c_{R}, k_{G_{l}}$, and $k_{l}$ are all non-negative, and hence [10]

$$
Q \cdot Q \geq-1, \quad Q \cdot Q+Q \cdot a \geq-2, \quad Q \cdot b_{G_{l}} \geq 0 .
$$

The left-moving central charge $c_{L}$ receives contributions from the left-moving current algebra associated with the flavor groups $G_{l}$ as

$$
c_{G}=\sum_{l} c_{G_{l}}:=\sum_{l} \frac{k_{G_{l}} \operatorname{dim}\left(G_{l}\right)}{k_{G_{l}}+h_{G_{l}}^{\vee}} .
$$

Here $h_{G_{l}}^{\vee}$ are the dual Coxeter numbers of $G_{l}$. Hence, the consistency of the worldsheet SCFT implies furthermore that $[10]$

$$
\sum_{l} c_{G_{l}} \leq c_{L}=3 Q \cdot Q-9 Q \cdot a+2 .
$$

This was used in Ref. [10] to rule out a number of theories with non-Abelian gauge groups, which would otherwise satisfy the 6D anomaly cancellation conditions.

If the $6 \mathrm{D}$ supergravity theory has a realization via $F$-theory compactified on an elliptic Calabi-Yau 3-fold $Y_{3}$ with projection

$$
\pi: Y_{3} \rightarrow B_{2},
$$

then the BPS strings correspond to solitonic strings from D3-branes wrapping a curve $C$ on the Kähler surface $B_{2}$. In this case, one identifies the string charge lattice with the cohomology lattice $H^{2}\left(B_{2}, \mathbb{Z}\right)$ and the intersection pairing with the cohomological intersection pairing of signature $(1, T)$. Furthermore, $-a=\bar{K}_{B_{2}}$ where $\bar{K}_{B_{2}}$ is the anticanonical class of $B_{2}$.

The condition for the string to not describe a 6D SCFT instanton string is that it wraps a curve of self-intersection not smaller than -1 . The worldsheet theory of such strings can then be obtained by reducing the four-dimensional (4D) $\mathcal{N}=4$ supersymmetric Yang-Mills theory on a single D3-brane along $C$ with the help of a topological duality twist [17], as detailed in Refs. [18,19]. The spectrum of worldsheet fields can be found in Table I. Suppose the curve class $C$ wrapped by a D3-brane allows for a decomposition $C=\sum_{p} n_{p} C_{p}$, where $C_{p}$ are the effective
TABLE I. Massless spectrum of the effective $2 \mathrm{D} \mathcal{N}=(0,4)$ worldsheet theory of the string that arises from a D3-brane wrapping a genus $g$ curve $C \subset B_{2}$ (not contained in the discriminant). The representations are under $S U(2)_{R} \times S U(2)_{l} \times$ $S O(1,1)$ and the $+/-$ signs in the second column denote chiralities.

\begin{tabular}{lcccc}
\hline \hline \multicolumn{1}{c}{ Fermions } & Bosons & $(0,4)$ & Multiplicity \\
\hline $2 \times(\mathbf{2}, \mathbf{1})_{1}$ & + & $4 \times(\mathbf{1}, \mathbf{1})_{0}$ & Hyper & $g-1+\bar{K}_{B_{2}} \cdot C$ \\
$2 \times(\mathbf{1}, \mathbf{2})_{1}$ & + & $(\mathbf{2}, \mathbf{2})_{0}$ & Twisted Hyper & 1 \\
$2 \times(\mathbf{1}, \mathbf{2})_{-1}$ & - & & Fermi & $g(C)$ \\
$(\mathbf{1}, \mathbf{1})_{-1}$ & - & & Half-Fermi & $8 \bar{K}_{B_{2}} \cdot C$ \\
\hline \hline
\end{tabular}

curve classes and $n_{p}>0$. Then, the 2D SCFT of the string associated with $C$ can split into the sum of SCFTs associated with $n_{p}$ D3-branes wrapping $C_{p}$ [18]. If the curve $C$ is irreducible, such a split occurs only for specially tuned values of the moduli of $C$, while for generic moduli the string flows to the SCFT associated with $C$. We will encounter such a situation in many cases. As long as the nondegenerate string associated with $C$ is itself not an SCFT instanton or LST string, bounds of the form (9) can be derived from it at generic points in the moduli space even though at special points in the moduli space the SCFT may degenerate to a sum of instanton strings.

\section{BOUNDS ON THE NUMBER OF $U(1)$ FACTORS IN SUPERGRAVITY}

We now derive bounds on the number of Abelian gauge group factors in $6 \mathrm{D} \mathcal{N}=(1,0)$ supergravity theories. We will be using the same constraint [Eq. (9)] as in Ref. [10], but applied to Abelian gauge groups.

The discussion can be phrased either in purely fieldtheoretic language or in the context of $F$-theory. We begin in this section with a supergravity approach. This, however, will only lead to bounds barring various assumptions concerning the integrality and nondegeneracy of suitable charge vectors, as we will make explicit. By contrast, in explicit string-theoretic realizations in $F$-theory (studied in the next section) no comparable assumptions have to be made, but these follow directly from the geometry of the compactification.

To each Abelian gauge group factor $U(1)_{i}, i=1, \ldots, N$, of a $6 \mathrm{D} \mathcal{N}=(1,0)$ supergravity we can associate an anomaly coefficient $b_{i}$ [5], which is the counterpart of the anomaly coefficient $b_{G_{l}}$ for the non-Abelian gauge groups. Similarly, each Abelian gauge group factor $U(1)_{i}$ induces a $U(1)_{i}$ current on the BPS string with charge vector $Q$, whose Kac-Moody level is given by

$$
k_{i}=Q \cdot b_{i},
$$

with the understanding that $k_{i}=0$ corresponds to no current. The $U(1)_{i}$ current central charge is 


$$
c_{i}:=c_{U(1)_{i}}=1 \text { for } k_{i} \neq 0 .
$$

Suppose now that the theory contains a BPS string with charge vector $Q$ subject to Eq. (7) such that for every $U(1)_{i}$ gauge group factor in the $6 \mathrm{D}$ supergravity theory the level on the string is positive,

$$
k_{i}=Q \cdot b_{i}>0 \quad \forall i=1, \ldots, N .
$$

Suppose furthermore that the associated string does not generically degenerate into SCFT instantons or LST strings. Then, the total number $N$ of $U(1)_{i}$ factors in the $6 \mathrm{D}$ supergravity theory is constrained to be

$$
N=\sum_{i} c_{i} \leq c_{L}=3 Q \cdot Q-9 Q \cdot a+2 .
$$

As we will discuss, a string charge vector $Q$ satisfying Eq. (13) can always be found as long as for each $U(1)_{i}$ there exists some hypermultiplet carrying nonzero $U(1)_{i}$ charge. Before showing this, note that the latter condition is always satisfied in theories with $T \leq 8$ [5]: recall that the anomaly coefficient $b_{i}$ appears, among other places, in the condition for the cancellation of the quartic $U(1)_{i}$ anomaly and the mixed $U(1)_{i}$-gravitational anomaly as

$$
\begin{aligned}
b_{i} \cdot b_{i} & =\frac{1}{3} \sum_{I} M_{I}\left(q_{I}^{(i)}\right)^{4}, \\
-a \cdot b_{i} & =\frac{1}{6} \sum_{I} M_{I}\left(q_{I}^{(i)}\right)^{2},
\end{aligned}
$$

where $M_{I}$ denotes the number of hypermultiplets with $U(1)_{i}$ charge $q_{I}^{(i)}$. Clearly, the first condition implies that $b_{i} \cdot b_{i} \geq 0$. If $b_{i} \cdot b_{i}=0$, then by Eq. (15) there is no charged matter, and one can furthermore show [8] that there can be no kinetic mixing with the remaining Abelian gauge factors $U(1)_{j}, j \neq i$. In this sense the $U(1)_{i}$ theory is completely trivial. In any case, theories with $b_{i} \cdot b_{i}=0$ cannot occur if $T \leq 8$ : the reason is that $b_{i} \cdot b_{i}=0$, together with $a \cdot b_{i}=0$ [which follows from Eqs. (15) and (16)] leads to $9-T=a \cdot a \leq 0$.

\section{A. Supergravities with $\boldsymbol{T}=\mathbf{0}$}

Let us now analyze the bound (14) on the number $N$ of $U(1)_{i}$ gauge group factors in supergravity. We begin with the simplest case, $T=0$. In this case $-a=3$ and $j=1$, and we consider the string with charge

$$
Q_{0}=1,
$$

with $Q_{0}^{2}=1$ and $Q_{0} \cdot a=-3$. Here and in the rest of this section we will always assume that the charge $Q_{0}$ is properly quantized so that a corresponding string satisfies the Dirac quantization condition; this is the first of a number of assumptions which can be manifestly verified in the $F$-theory realizations presented in the next section. According to the completeness conjecture [20] all strings on the charge lattice are in the physical spectrum of a quantum gravity theory, and hence there exists a string with charge (17).

Since $b_{i}^{2}>0$ and $j \cdot b_{i}>0$, we know that $b_{i}>0$ and hence $Q_{0} \cdot b_{i}>0$. It is furthermore clear that the string cannot degenerate to a product of SCFT or LST strings since there are no such sectors in a theory with no tensor multiplets. Therefore, the number of $U(1)_{i}$ factors is bounded as

$$
N \leq c_{L}=32, \quad T=0 .
$$

This bound is to be compared with the conditional bound $N \leq 17$ derived previously in Ref. [5] based entirely on the 6D supergravity anomaly conditions. We emphasize that our bound is universal for $T=0$ supergravities and in particular also works in the presence of a non-Abelian sector.

\section{B. Supergravities with $T=1$}

Assume next that $T=1$. There exist two choices for the unimodular charge lattice [15],

$$
\Gamma_{0}=\left(\begin{array}{ll}
0 & 1 \\
1 & 0
\end{array}\right) \quad \text { or } \quad \Gamma_{1}=\left(\begin{array}{cc}
1 & 0 \\
0 & -1
\end{array}\right)
$$

Let us first discuss $\Gamma_{1}$ : one can always choose, without loss of generality, $-a=(3,-1)$ [4]. We consider the string with charge

$$
Q_{0}=(1,-1),
$$

which satisfies

$$
Q_{0} \cdot Q_{0}=0, \quad Q_{0} \cdot a=-2
$$

and therefore fulfills the first two conditions in Eq. (7). Let us furthermore assume that the string with charge $Q_{0}$ does not degenerate (generically) into several strings, including instanton strings. We will explicitly verify this in the $F$ theory realization of these models in the next section, but for now we leave it as an assumption from the pure supergravity perspective. Let us make the ansatz

$$
b_{i}=\left(b_{i}^{0}, b_{i}^{1}\right) .
$$

The condition $b_{i} \cdot b_{i}>0$, known to be valid for $T=1$, enforces that

$$
b_{i}^{0}>\left|b_{i}^{1}\right| \geq 0 \quad \text { or } \quad b_{i}^{0}<-\left|b_{i}^{1}\right| \leq 0 .
$$

The second possibility can be excluded by recalling that $j \cdot b_{i}$ denotes the diagonal part of the matrix involving the 
Abelian gauge kinetic couplings and hence must be nonnegative whenever $j$ lies in the cone of allowed tensor multiplet vacuum expectation values. Let us approach the boundary of this cone (subject to the normalization $j \cdot j=1)$ by sending $j \rightarrow(1,0)$. Then, demanding that $j \cdot b_{i}$ remains non-negative rules out the case $b_{i}^{0}<0$ and thereby the second option in Eq. (23). We thus conclude that

$$
k_{i}=Q_{0} \cdot b_{i}=b_{i}^{0}+b_{i}^{1}>0 .
$$

This means that each $U(1)_{i}$ couples to the string, and therefore demanding Eq. (14) implies that the number $N$ of $U(1)_{i}$ factors in the supergravity theory is bounded as

$$
N \leq c_{L}=20, \quad(T=1) .
$$

For the lattice $\Gamma_{0}$, there exist two inequivalent choices for $a$ subject to $a \cdot a=8$, namely, $-a=(2,2)$ or $-a=(4,1)$ [15]. Either way, $b_{i} \cdot b_{i}>0$ implies that in $b_{i}=\left(b_{i}^{0}, b_{i}^{1}\right)$ both entries must be nonvanishing and hence $Q \cdot b_{i} \neq 0$ for any $Q=\left(Q^{0}, Q^{1}\right)$. If $-a=(2,2)$, one then obtains the same bound (25) for $Q_{0}=(1,0)$ with $Q_{0} \cdot Q_{0}=0$ and $Q_{0}$. $(-a)=2$ and with the convention for the cone of scalar fields $j$ that $b_{i}^{1}>0$ to satisfy $j \cdot b_{i}>0$. If $-a=(4,1)$, this choice would imply $Q_{0} \cdot Q_{0}=0$ and $Q_{0} \cdot(-a)=1$. If this $Q_{0}$ is properly quantized, it is clear that this cannot have a geometric interpretation in terms of a curve class on a Kähler surface in an $F$-theory context (for which the Riemann-Roch theorem would have to hold). Indeed, the choice $-a=(4,1)$ does not have a known string-theoretic realization [6]. In any event, we would obtain the bound $N \leq 11$ from $Q_{0}$ for $-a=(4,1)$, and by comparison with Eq. (25), $N \leq 20$ remains to be the relevant constraint for $T=1$ supergravities.

We will give an interpretation of this constraint in the next section. Furthermore, it is interesting to note that Eq. (25) is much stronger than the bound

$N \leq(T+2)\left(T+\frac{7}{2}+\left(T^{2}-51 T+\frac{2225}{4}\right)^{1 / 2}\right)$,

which was found in Ref. [5] for a theory with only Abelian gauge group factors and $T \leq 8$ : for $T=1$, Eq. (26) merely constrains the number of Abelian gauge group factors to be $N \leq 81$.

\section{Supergravities with $T>1$}

It is clear that even for $1<T \leq 8$ these results can be generalized, where for simplicity we only consider supergravity models with charge lattice

$\Gamma=\operatorname{diag}(1,-1, \ldots,-1), \quad-a=(3,-1, \ldots,-1)$.

Consider the string with charge

$$
Q_{0}=(1,-1,0, \ldots, 0)
$$

and make the ansatz $b_{i}=\left(b_{i}^{0}, b_{i}^{1}, b_{i}^{2}, \ldots, b_{i}^{T}\right)$. Again, $b_{i}$. $b_{i}>0$ implies Eq. (23), and the second option can be excluded by demanding $j \cdot b_{i}>0$ for the boundary value $j=(1,0, \ldots, 0)$. Hence, Eq. (24) continues to hold, barring the assumption that $Q_{0}$ is an integer charge vector corresponding to a nondegenerate string.

A similar analysis, again subject to the assumptions concerning the integrality and nondegeneracy of charge vectors, can also be carried out for supergravities with $T \geq 9$ and Eq. (27). If $b_{i} \cdot b_{i} \neq 0$ for every $U(1)_{i}$ factor, the same bound (25) applies as in the theories with $1 \leq T \leq 8$ and $-a=(3,-1, \ldots,-1)$. Even in the presence of $b_{i}$ 's with $b_{i} \cdot b_{i}=0$, a relaxed bound of 22 can be obtained by considering $T$ strings with charges

$Q_{0}^{(\alpha)}=(1,0, \ldots, 0,-1,0, \ldots, 0), \quad$ for $\alpha=1, \ldots, T$,

for which the only nonzero entries are the zeroth and the $\alpha$ th components: upon combining the resulting constraints, one can bound the total number of $U(1)$ factors by

$$
N \leq 20 \frac{T}{T-1} \leq 22.5
$$

where $T \geq 9$ has been used in the last step. In particular, this allows us to rule out models with an infinite number of Abelian gauge groups with $b_{i} \cdot b_{i}=0$. As shown in Ref. [5], this possibility cannot be ruled out based solely on the 6D supergravity anomaly cancellation conditions.

In summary, for the charge lattice choices made above, the consistency conditions from BPS strings lead to a considerably stronger bound on the number of Abelian gauge group factors than $6 \mathrm{D}$ anomaly considerations alone.

\section{BOUNDS ON THE NUMBER OF $U(1)$ FACTORS IN $\boldsymbol{F}$-THEORY}

We now interpret and sharpen the bounds found in the previous section, focusing on those supergravity theories with a realization as an $F$-theory compactification on an elliptic Calabi-Yau 3-fold $\pi: Y_{3} \rightarrow B_{2}$. In order to avoid nonminimal singularities in the fiber, which would spoil the Calabi-Yau condition, the base $B_{2}$ of the elliptic fibration must be of one of the following three types [22]: ${ }^{1}$

(1) $B_{2}=\mathbb{P}^{2} \Rightarrow T=0$.

(2) $B_{2}=\mathbb{F}_{n}$ with $n=0, \ldots, 8,12 \Rightarrow T=1$.

(3) $B_{2}=\mathrm{Bl}^{k}\left(\mathbb{F}_{n}\right)$, a $k$-fold blowup of $\mathbb{F}_{n} \Rightarrow T=1+k$. In this paper, whenever we speak of $F$-theory constructions we specifically have one of these three smooth classes of base spaces in mind.

\footnotetext{
${ }^{1}$ In addition, the F-theory base could be an Enriques surface; in this case, there are no gauge fields in F-theory [21] and we hence do not consider this choice further.
} 
Abelian gauge potentials $A_{i}$ in the effective action arise by expanding the M-theory 3 -form $C_{3}$ as $C_{3}=\sum_{i} A_{i} \wedge \mathrm{w}_{i}$, where $\mathrm{w}_{i} \in H^{2}\left(Y_{3}, \mathbb{R}\right)$ is the image of a rational section $S_{i}$ of $Y_{3}$ under the so-called Shioda map, $\mathrm{w}_{i}=\sigma\left(S_{i}\right)$. The rational sections of $Y_{3}$ form the Mordell-Weil group $\operatorname{MW}\left(Y_{3}\right)$, whose rank therefore counts the number of independent (non-Cartan) $U(1)_{i}$ gauge factors in the effective action. See Refs. [23,24] and references therein for more information.

The anomaly coefficients $b_{i}$ associated with such $U(1)_{i}$ gauge factors are given by the so-called height pairing

$$
b_{i}=-\pi_{*}\left(\sigma\left(S_{i}\right) \cdot \sigma\left(S_{i}\right)\right) .
$$

The crucial property which we will be using is that, as discussed in Ref. [8] based on the considerations in Ref. [25], this divisor class can always be written as

$$
b_{i}=\frac{1}{m_{i}^{2}}\left(2 \bar{K}_{B_{2}}+\delta_{i}\right), \quad \text { for some } m_{i} \in \mathbb{Z}_{>0},
$$

where $\delta_{i}$ is an effective divisor on $B_{2}$ and $\bar{K}_{B_{2}}$ represents the anticanonical class on $B_{2}$. The effective divisor $b_{i}$ can be thought of as the linear combination of 7-brane divisors supporting the Abelian gauge group $U(1)_{i}$.

We can now interpret and sharpen the constraints found in the previous section for the three types of $F$-theory base spaces listed above.

\section{A. $F$-theory vacua with $T=0$}

For $T=0$, i.e., $B_{2}=\mathbb{P}^{2}$, we can consider a string by wrapping a D3-brane along the rational curve $C=H$, where $H$ denotes the hyperplane class of $B_{2}$ with $H \cdot H=1$, in terms of which $\bar{K}_{\mathbb{P}^{2}}=3 H$. Every height pairing $b_{i}$ can be parametrized as $b_{i}=b H$ for $b>0$. This system is of course an explicit realization of the supergravity setup with $T=0$ considered in the previous section and simply reproduces the bound (18). We will come back to this setup in Sec. V and argue that this bound can be sharpened.

\section{B. $F$-theory vacua with $T=1$}

For $T=1$, the $F$-theory base is a Hirzebruch surface $B_{2}=\mathbb{F}_{n}$, for $n=0,1, \ldots, 8,12$, corresponding to a fibration of a rational curve $\mathbb{P}_{f}^{1}$ over a base $\mathbb{P}_{h}^{1}$ [26]. The classes of the fiber $f$ and of the base $h$ span the cohomology $H^{2}\left(\mathbb{F}_{n}, \mathbb{Z}\right)=\langle f, h\rangle$ and have the intersection numbers

$$
f \cdot f=0, \quad f \cdot h=1, \quad h \cdot h=-n .
$$

The Mori cone of effective curves $\mathbf{M}\left(\mathbb{F}_{n}\right)$ and the closure of the Kähler cone $\overline{\mathbf{K}}\left(\mathbb{F}_{n}\right)$ are given by

$$
\mathbf{M}\left(\mathbb{F}_{n}\right)=\langle f, h\rangle, \quad \overline{\mathbf{K}}\left(\mathbb{F}_{n}\right)=\langle f, h+n f\rangle,
$$

while the anticanonical class evaluates to

$$
\bar{K}_{\mathbb{F}_{n}}=2 h+(2+n) f .
$$

Consider now the string obtained by wrapping a D3-brane along the curve class

$$
C=f
$$

with

$$
C \cdot C=0, \quad C \cdot \bar{K}_{\mathbb{F}_{n}}=2 .
$$

This is an explicit realization of the properties (21) for an integral curve class $C$. Since $C=f$ is a generator of the Mori cone, it cannot split into other effective curve classes. The worldsheet SCFT associated with the string is therefore manifestly nondegenerate. Since $f$ is furthermore in the closure of the Kähler cone (34), we know that

$$
f \cdot \delta \geq 0 \quad \forall \delta \text { effective. }
$$

As a result, any $U(1)_{i}$ factor in the theory leads to a worldsheet current on the string with a nontrivial KacMoody level because

$k_{i}=C \cdot b_{i}=f \cdot \frac{1}{m_{i}^{2}}\left(2 \bar{K}_{\mathbb{F}_{n}}+\delta_{i}\right) \geq \frac{2}{m_{i}^{2}} f \cdot \bar{K}_{\mathbb{F}_{n}}=\frac{4}{m_{i}^{2}}>0$,

where we used Eq. (32). Hence, the string associated with $C$ satisfies all properties to apply Eq. (14) to bound the number $N$ of Abelian gauge group factors in such $F$-theory models, leading again to the bound

$$
N \leq c_{L}=20 .
$$

In fact, the string from the D3-brane wrapped on $C=f$ is not just any solitonic string: its zero-mode spectrum coincides with the spectrum of a critical heterotic string propagating in six dimensions; see Table I for the field contents of the worldsheet theory. This is of course no surprise because $F$-theory on $B_{2}=\mathbb{F}_{n}$ is dual to the heterotic string on an elliptic $K 3$ over $\mathbb{P}_{h}^{1}$, and in this duality the solitonic string from a D3-brane wrapping the fiber $\mathbb{P}_{f}^{1}$ reduces to the critical heterotic string in the limit of small volume of $\mathbb{P}_{f}^{1}$. This realization of the heterotic string has played an important role in the context of the weak gravity conjecture in $F$-theory [11-14].

The value $c_{L}=20$ of the heterotic string has an immediate interpretation: the left-moving sector of the string (apart from the free hypermultiplet associated with the string motion in $\mathbb{R}^{1,5}$ ) comprises $8 C \cdot \bar{K}_{\mathbb{F}_{n}}=16$ leftmoving fermions in $\mathcal{N}=(0,4)$ half-Fermi multiplets, along with one hypermultiplet associated with the propagation of the string on the dual heterotic K3 surface 
(see Table I). The half-Fermi multiplets are due to the zero modes at the intersection of $C$ with the 7-branes on $B_{2}$. They are the only source of charge of the string with respect to the 7-brane gauge groups since the hypermultiplet is uncharged. This suggests that the number of $U(1)_{i}$ gauge fields is in fact subject to the slightly stronger bound

$$
N \leq\left(c_{L}-4\right)=16
$$

where we have subtracted the contribution from the $\left[U(1)_{i}\right.$ neutral] hypermultiplet associated with the moduli of the string along the dual K3 surface. In hindsight, the bound (41) is simply the well-known statement that the $U(1)_{i}$ gauge groups in models with a perturbative heterotic dual on a smooth $K 3$ can be embedded into the perturbative heterotic $E_{8} \times E_{8}$ gauge group. A far less trivial statement is that the bound $N \leq 16$ continues to hold in all $F$-theory compactifications with $T>1$, as we will see momentarily.

It is interesting to compare the bound (41) relevant for geometric $F$-theory vacua to the less strict bound (25) derived for general supergravity theories with $T=1$. Consistent supergravities satisfying Eq. (25) but not Eq. (41) exist. For instance, $6 \mathrm{D} \mathcal{N}=(1,0)$ vacua of the perturbative heterotic string can have a gauge group of rank up to 20 , as is known from purely conformal field-theoretic constructions (e.g., Ref. [28]). These are, however, not the geometric heterotic K3 compactifications which the solitonic string in our $F$-theory model is related to [29]. Correspondingly, a split of the worldsheet fields of a heterotic string as in Table I, which underlies the reduced bound (41), is a consequence of the specific realization of the heterotic worldsheet theory as a wrapped D3-brane in $F$-theory setups. In this sense, the bound $N \leq 20$ found in the supergravity approach, without any prejudice concerning an embedding into $F$-theory, is indeed the correct bound for general $6 \mathrm{D} \mathcal{N}=(1,0)$ supergravities.

Finally, recall the well-known fact that $F$-theory on $\mathbb{F}_{n}$ necessarily supports a non-Higgsable non-Abelian gauge group $G_{n}$ on 7-branes wrapping the base class $h$ with selfintersection $h \cdot h=-n$. Since $b_{G_{n}} \cdot C=h \cdot f=1$, this gives rise to a $G_{n}$ current on the string at level $k_{G_{n}}=1$, which contributes to the central charge $c_{L}$ with $c_{G_{n}}$ given in Eq. (8). From Table II, we find that this improves the bound on the number of possible Abelian gauge group factors to be

$N \leq\left\lfloor 16-c_{G_{n}}\right\rfloor=\{16,16,16,14,12,10,10,9,9,8\}$

TABLE II. Non-Higgsable gauge groups on $\mathbb{F}_{n}$.

\begin{tabular}{ccccccccccc}
\hline \hline $\mathbb{F}_{n}$ & 0 & 1 & 2 & 3 & 4 & 5 & 6 & 7 & 8 & 12 \\
\hline$G_{n}$ & - & - & - & $S U(3)$ & $S O(8)$ & $F_{4}$ & $E_{6}$ & $E_{7}$ & $E_{7}$ & $E_{8}$ \\
$h_{G}^{\vee}$ & - & - & - & 3 & 6 & 9 & 12 & 18 & 18 & 30 \\
\hline \hline
\end{tabular}

for $F$-theory models on $B_{2}=\mathbb{F}_{n}$ with $n=0,1, \ldots, 8,12$, respectively.

\section{C. $F$-theory vacua with $T>1$}

We now come to the most interesting case, where the $F$-theory base $B_{2}$ is an arbitrary blowup of $\mathbb{F}_{n}$. Recall that $\mathbb{F}_{n}$ is a nondegenerate $\mathbb{P}_{f}^{1}$ fibration. After we blow up a single point into an exceptional divisor (curve), the new fiber degenerates into two curves when it hits the blowup locus. This process can be repeated multiple times. Let us introduce the morphism

$$
p: B_{2} \rightarrow \mathbb{F}_{n}
$$

which corresponds to the blowdown of the exceptional divisors $E_{l}$ on $B_{2}$. The anticanonical classes of both spaces are related as $\bar{K}_{B_{2}}=p^{*}\left(\bar{K}_{\mathbb{F}_{n}}\right)+\sum_{l} a_{l} E_{l}$ for some coefficients $a_{l}$. The idea is now to consider the string obtained from a D3-brane wrapping the curve

$$
C=p^{*}(f) \subset B_{2},
$$

where $f$ is the fiber class on $\mathbb{F}_{n}$ prior to the blowup. This curve has the properties

$$
C \cdot{ }_{B_{2}} C=p^{*}(f) \cdot_{B_{2}} p^{*}(f)=f \cdot{ }_{\mathbb{F}_{n}} f=0
$$

and

$$
\begin{aligned}
C \cdot{ }_{B_{2}} \bar{K}_{B_{2}} & =p^{*}(f) \cdot B_{B_{2}}\left(p^{*}\left(\bar{K}_{\mathbb{F}_{n}}\right)+\sum_{l} a_{l} E_{l}\right) \\
& =f \cdot_{\mathbb{F}_{n}} \bar{K}_{\mathbb{F}_{n}}+\sum_{l} a_{l} f \cdot \mathfrak{F}_{n} p_{*}\left(E_{l}\right) \\
& =f \cdot \cdot_{\mathbb{F}_{n}} \bar{K}_{\mathbb{F}_{n}}=2 .
\end{aligned}
$$

Before the last line we have used that $f{ }_{\mathfrak{F}_{n}} p_{*}\left(E_{l}\right)=0$ because the blowdown map $p$ contracts the exceptional divisors to points.

The string associated with $C$ therefore still has the same zero-mode structure as the heterotic string in six dimensions. As alluded to above, the difference with the situation with $T=1$ is that the curve $C=p^{*}(f)$ may split, at certain loci in its moduli space, into various exceptional divisors on $B_{2}$. In the dual heterotic picture, this corresponds to a degeneration of the heterotic string into various noncritical strings at the location of NS5-branes. Importantly for us, away from the NS5-branes, the heterotic worldsheet is nondegenerate and continues to be described by a single SCFT in the IR for which the analysis of Sec. II is valid.

It is still true that each $U(1)_{i}$ gauge symmetry in the $6 \mathrm{D}$ $F$-theory induces an Abelian worldsheet current on the string of nonvanishing level $k_{i} \neq 0$. This is because prior to 
the blowup, $f$ is in the closure $\overline{\mathbf{K}}\left(\mathbb{F}_{n}\right)$ of the Kähler cone, and its pullback under the blowdown (43) continues to lie in the Kähler cone closure [30]. Therefore, the same computation as in Eq. (39), now performed on $B_{2}$, shows that

$$
k_{i}=C \cdot_{B_{2}} b_{i}>0 \quad \forall b_{i} .
$$

We conclude that the same upper bound $N \leq c_{L}=20$, or in fact

$$
N \leq\left(c_{L}-4\right)=16
$$

holds for any $F$-theory compactification with at least one tensor [or in fact the stronger bound (42) for $F$-theory on a $k$-fold blowup of $\left.\mathbb{F}_{n}\right]$. Here we have again subtracted the contribution from the $U(1)_{i}$ uncharged hypermultiplet on the worldsheet of the heterotic string as realized by a wrapped D3-brane.

Note that this certainly does not mean that the total rank of the gauge group, including non-Abelian factors, should be bounded by 16: unlike Abelian gauge group factors, non-Abelian gauge symmetries can "hide" from the worldsheet of the heterotic string in that they need not necessarily induce a worldsheet current with nonzero level $k_{G_{i}}$. After all, the crucial property of Abelian gauge symmetries underlying our analysis is that their anomaly coefficients take the form (32), which prevents $k_{i}=0$ for the heterotic string under consideration. Non-Abelian gauge group factors with $k_{G_{l}}=0$ with respect to the heterotic string are truly nonperturbative in that they cannot be embedded into the heterotic $E_{8} \times E_{8}$ current on the heterotic worldsheet. The anomaly coefficients of such gauge groups satisfy

$$
b_{G_{l}} \cdot b_{G_{l}} \leq 0
$$

because $b_{G_{t}} \cdot C=0$ and $C \cdot C=0$ can only be solved, with an intersection form of signature $(1, T)$, for $b_{G_{l}} \cdot b_{G_{l}} \leq 0$ (and $b_{G_{l}} \cdot b_{G_{l}}=0$ if and only if $b_{G_{l}}=\alpha C$ for some $\alpha \in \mathbb{Q}$ ). In fact, $b_{G_{t}}$ is the divisor class wrapped by the 7-brane with gauge group $G_{l}$, and the gauge groups with $b_{G_{l}} \cdot C=0$ include all the gauge groups wrapping blowup divisors on $B_{2}$ : as discussed, these blowup divisors are contained in $C=p^{*}(f)$ and hence intersect it trivially. These exceptional divisors support 6D SCFT sectors on shrinkable curves on $B_{2}$ and are, in the above sense, truly nonperturbative from the heterotic string point of view [31]. In particular, our results imply that it is not possible to Higgs these to Abelian gauge group factors. This is (trivially) true not only for the non-Higgsable gauge groups supported on blowup divisors, but also after enhancing the gauge group further by tunings of the elliptic fibration in $F$-theory. Even after such tunings that enhance $G \rightarrow H$, there are no adjoint scalars available to Higgs $H \rightarrow G \times U(1)^{l}$ because the blowup curves are rigid.

Another way to phrase the origin of the bound (50) is this: $F$-theory on a blowup of $\mathbb{F}_{n}$ is dual to the heterotic string on K3 with NS5-branes. Due to nonperturbative effects associated with the NS5-branes, additional gauge groups will in general occur which cannot be embedded into $E_{8} \times E_{8}$. In $F$-theory these are realized on the blowup divisors, and since the latter have vanishing intersection with the curve $C=p^{*}(f)$ they do not correspond to a current algebra on the heterotic string. On the other hand, Abelian gauge groups are guaranteed to induce a $U(1)_{i}$ current on the worldsheet because of Eq. (49). Hence, the nonperturbative sector does not contain the $U(1)_{i}$ gauge groups, which are consequently purely perturbative and embeddable into $E_{8} \times E_{8}$.

\section{DISCUSSION AND SPECULATIONS}

In this short paper, we have derived universal bounds on the number $N$ of Abelian gauge group factors in $F$-theory compactifications to six dimensions. Our main result is the bound $N \leq 16$ for any $6 \mathrm{D} F$-theory compactification giving rise to at least one tensor multiplet. The derivation combines the recent idea of Ref. [10] to deduce constraints on the form of $6 \mathrm{D} \mathcal{N}=(1,0)$ supergravity theories from the consistency of embedded BPS strings on the one hand, and specific properties of the anomaly coefficients for Abelian gauge group factors $[8,25]$ on the other hand. As in Refs. [11-14], we have identified a certain solitonic string on any $F$-theory base other than $\mathbb{P}^{2}$ as the heterotic string; applying the constraint (9) to this string in the context of Abelian gauge groups leads to the precise bounds (42) and (50). For $F$-theory on an elliptic fibration over base $\mathbb{P}^{2}$, no such heterotic string can be identified in the spectrum of solitonic strings; the resulting bound found for this class of models hence a priori only takes the form $N \leq 32$. We will come back to this point below.

Our results are interesting from the perspective of the swampland program of ruling out theories without a UV completion despite their (apparent) low-energy consistency; e.g., in Ref. [5] it was observed that 6D anomaly cancellation alone is not enough to rule out 6D supergravity models with an infinite number of $U(1)$ gauge fields with anomaly coefficients $b_{i} \cdot b_{i}=0$. Our analysis shows that such theories are in the swampland, both from the perspective of $F$-theory and-barring the assumptions stated-more generally in supergravity even without invoking any string-theoretic realization.

Our results can also be interpreted as giving a prediction for the maximal rank of the Mordell-Weil group of any elliptically fibered Calabi-Yau 3-fold to be

$\operatorname{rk}\left(\mathrm{MW}\left(Y_{3}\right)\right) \leq 16\left(B_{2} \neq \mathbb{P}^{2}\right)$ or $\leq 32\left(B_{2}=\mathbb{P}^{2}\right)$, 
where the bound for $B_{2}=\mathbb{P}^{2}$ will be revisited below. To date, the elliptically fibered Calabi-Yau 3-fold with the highest known Mordell-Weil rank is an elliptic fibration over $\mathbb{P}^{2}$ with $\operatorname{rk}\left(\mathrm{MW}\left(Y_{3}\right)\right)=10$ [32]. It would be interesting to see if the bounds (52) for elliptic Calabi-Yau 3-folds can be improved further, or else be derived from a purely mathematical point of view.

We end with a number of extensions of our results, some of which are speculative.

\section{A. $F$-theory on $\mathbb{P}^{2}$ revisited}

First, it is natural to conjecture that the universal bound $N \leq 16$ should also apply to $F$-theory with base $\mathbb{P}_{2}$. The total value of $c_{L}=32$ for the string associated with $C=H$ on $\mathbb{P}^{2}$ can be understood as $c_{L}=24+8$, where the second factor is due to the two interacting hypermultiplets of the string (see again Table I) and the first factor is due to the $8 \bar{K}_{\mathbb{P}^{2}} \cdot C=24$ fermionic 3-7 modes. Since these are the only source of $U(1)_{i}$ charge of the string, it is again suggestive that $N \leq c_{L}-8=24$.

However, we conjecture that this is still an overcounting. To understand its origin in our framework, note that the surface $\mathbb{P}^{2}$ can be obtained from $\mathbb{F}_{1}$ by blowing down the base curve $\mathbb{P}_{h}^{1}$ with class $h$. On $\mathbb{F}_{1}$, we can consider the string associated with the curve class $C^{\prime}=f+h$. By blowing down $h, C^{\prime}$ maps to the class $C=H$ associated with the string on $\mathbb{P}^{2}$ that we are interested in. By wrapping a D3-brane along $C^{\prime}$ on $\mathbb{F}_{1}$ we obtain a bound state of the heterotic string (from a D3-brane on $f$ ) with one E-string (from a D3-brane on $h$ with $h \cdot h=-1$ ). At generic values of the curve moduli the string is nondegenerate and described by the invariants $C^{\prime} \cdot C^{\prime}=1, C^{\prime} \cdot \bar{K}_{\mathbb{F}_{1}}=3$. This matches the string on the curve $C=H$ after the blowdown on $\mathbb{P}^{2}$. The total left-moving central charge for the string on $C^{\prime}$ is $c_{L}=32=24+8$. Since $C^{\prime}=h+f$ is in the Kähler cone (34), we know that $k_{i}=b_{i} \cdot C^{\prime} \neq 0$ for every $U(1)_{i}$ in the theory, but $b_{i} \cdot h$ need not be nonvanishing. Indeed, the heterotic string has a current algebra $E_{8}^{(1)} \times E_{8}^{(2)}$, while the $E$-string has only a single $E_{8}$ current, and some $U(1)_{i}$ factors are orthogonal to this $E_{8}$. The bound $N \leq 24$ can then be interpreted as $N \leq N_{f}+N_{h}$, where $N_{f}=16$ is the number of $U(1)_{i}$ that couple to the heterotic string and $N_{h}$ is the number of $U(1)_{i}$ coupling to the E-string. Since the latter set is included in the first, we end up with $N \leq 16$ for the total number of $U(1)_{i}$. Now, on $\mathbb{P}^{2}$, there exists no individual heterotic and $E$-string, but only their bound state along $C=H$; nonetheless, we conjecture that a similar overcounting is at work, and that the bound can be improved to $N \leq 16$, which is even sharper than the conditional bound $N \leq 17$ from anomalies [5]. This translates into the conjecture that $\operatorname{rk}\left(\operatorname{MW}\left(Y_{3}\right)\right) \leq$ 16 for any elliptic Calabi-Yau 3-fold with base $\mathbb{P}^{2}$, and it would be extremely interesting to verify or falsify this speculation.

\section{B. Extensions to 4D $F$-theory}

Our findings can be immediately extended to $F$-theory compactifications on Calabi-Yau 4-folds to four dimensions. This is particularly useful because no comparable anomaly constraints exist in this case, at least for (nonchiral) situations without gauge fluxes.

Strings from D3-branes wrapping curves $C$ on the base 3 -fold $B_{3}$ are now described by a $2 \mathrm{D} \mathcal{N}=(0,2)$ worldsheet theory [19], even though these strings are not BPS objects from the viewpoint of the $4 \mathrm{D} \mathcal{N}=1$ supersymmetry algebra. As long as $B_{3}$ is the blowup of a $\mathbb{P}_{f}^{1}$ fibration, a D3-brane wrapping the class of the generic $\mathbb{P}_{f}^{1}$ fiber gives rise to a heterotic string, which becomes the fundamental string in the dual heterotic frame [13]. This continues to hold even if no perturbative heterotic dual of the $F$-theory model exists due to the presence of NS5branes on the heterotic side. These now wrap curves on the base of the dual heterotic Calabi-Yau 3-fold, which are the location over which the blowups have been performed on the $F$-theory side. The form (32) for the $U(1)_{i}$ anomaly coefficients $b_{i}=\frac{1}{m_{i}^{2}}\left(2 \bar{K}_{B_{3}}+\delta_{i}\right)$ carries over to Calabi-Yau 4-folds and by similar arguments as before, every $U(1)_{i}$ couples to the heterotic worldsheet in $F$-theory. More precisely, prior to the blowup, let us denote by $r: B_{3} \rightarrow$ $B_{2}$ the $\mathbb{P}_{f}^{1}$ fibration of $B_{3}$, where $B_{2}$ is a surface; then, we can always realize the fiber $\mathbb{P}_{f}^{1}$ as the class $\mathbb{P}_{f}^{1}=\frac{1}{n}\left(r^{*} J_{0}\right.$. $r^{*} J_{0}$ ) for some Kähler form $J_{0}$ on $B_{2}$ (where $n$ is an integer). Now, $r^{*} J_{0} \cdot r^{*} J_{0} \cdot \delta_{i} \geq 0$ because $J_{0}$ pulls back to an element in the closure of the Kähler cone of $B_{3}$ and $\delta_{i}$ is effective, and $r^{*} J_{0} \cdot r^{*} J_{0} \cdot \bar{K}_{B_{3}}=2$ because the fiber is a rational curve of self-intersection zero. Hence, $k_{i}=b_{i} \cdot\left[\mathbb{P}_{f}^{1}\right] \geq 4 / m_{i}^{2}>0$. This result remains invariant under blowups of $B_{3}$.

We therefore reproduce the same bound

$$
N \leq c_{L}-6=22-6=16
$$

where $c_{L}=22$ denotes the left-moving central charge of the internal sector (after subtracting the contribution from two free scalars corresponding to the motion in the two transverse directions) and the subtraction of six is for the chiral left-moving scalars which are uncharged under the 7-brane gauge group, as before. See Table 3.1. of Ref. [13] for details of the worldsheet spectrum.

Note that Eq. (53) only constrains the number of Abelian gauge group factors, which are realized in terms of rational sections, on blowups of rational fibrations. Unlike in six dimensions, even the part of the non-Abelian gauge sector which is not embedded in the heterotic worldsheet algebra may contribute additional $U(1)$ 's due to the possibility of breaking the gauge group with gauge fluxes. These sectors are not constrained by our arguments. Such $U(1)$ 's, however, are not realized by rational sections of the fibration. 
Furthermore, the set of possible base spaces is considerably richer for four-dimensional $F$-theory, and it would be interesting to extend our bounds to other geometries.

\section{Constraints on charges}

Coming back to six dimensions for simplicity, the essence of our bounds is the realization that Abelian gauge groups are embedded into the $E_{8} \times E_{8}$ algebra of a heterotic worldsheet, at least for models with $T \geq 1$. This statement holds independently of the existence of a perturbative heterotic dual. It should also carry over to the level of charged matter states in that not only is the possible number of Abelian gauge group factors constrained, but also the possible spectrum of charges. In other words, all
Abelian gauge charges in $F$-theory models with $T \geq 1$ should follow from a decomposition of the $\mathbf{2 4 8} \times \mathbf{2 4 8}$ of $E_{8} \times E_{8}$. It would be very interesting to understand this further, in particular in view of recent attempts to classify possible charges in $F$-theory geometry (e.g., in Refs. [6,33-36], with a more complete list of references provided in Refs. $[23,24])$.

\section{ACKNOWLEDGMENTS}

We thank Wolfgang Lerche for crucial discussions and for collaboration on related topics. The work of S. J. L. is supported by the Korean Research Foundation (KRF) through the CERN-Korea Fellowship program.
[1] C. Vafa, arXiv:hep-th/0509212.

[2] T. D. Brennan, F. Carta, and C. Vafa, Proc. Sci., TASI2017 (2017) 015.

[3] E. Palti, Fortschr. Phys. 67, 1900037 (2019).

[4] V. Kumar, D. R. Morrison, and W. Taylor, J. High Energy Phys. 11 (2010) 118.

[5] D. S. Park and W. Taylor, J. High Energy Phys. 01 (2012) 141.

[6] W. Taylor and A. P. Turner, J. High Energy Phys. 06 (2018) 010 .

[7] W. Taylor, arXiv:1104.2051.

[8] S. J. Lee, D. Regalado, and T. Weigand, J. High Energy Phys. 11 (2018) 147.

[9] Ref. [5] also establishes another bound on the number of $U(1)$ factors in presence of non-Abelian gauge sectors, which depends in addition on the number of non-abelian matter representations.

[10] H. C. Kim, G. Shiu, and C. Vafa, arXiv:1905.08261.

[11] S. J. Lee, W. Lerche, and T. Weigand, J. High Energy Phys. 10 (2018) 164.

[12] S. J. Lee, W. Lerche, and T. Weigand, Nucl. Phys. B938, 321 (2019).

[13] S. J. Lee, W. Lerche, and T. Weigand, arXiv:1901.08065.

[14] S. J. Lee, W. Lerche, and T. Weigand, arXiv:1904.06344.

[15] N. Seiberg and W. Taylor, J. High Energy Phys. 06 (2011) 001.

[16] F. Benini and N. Bobev, J. High Energy Phys. 06 (2013) 005.

[17] L. Martucci, J. High Energy Phys. 06 (2014) 180.

[18] B. Haghighat, S. Murthy, C. Vafa, and S. Vandoren, J. High Energy Phys. 01 (2016) 009.

[19] C. Lawrie, S. Schäfer-Nameki, and T. Weigand, J. High Energy Phys. 04 (2017) 111.
[20] J. Polchinski, Int. J. Mod. Phys. A 19, 145 (2004).

[21] J. D. Blum and A. Zaffaroni, Phys. Lett. B 387, 71 (1996).

[22] A. Grassi, Math. Ann. 290, 287 (1991).

[23] T. Weigand, Proc. Sci., TASI2017 (2018) 016.

[24] M. Cvetič and L. Lin, Proc. Sci., TASI2017 (2018) 020.

[25] D. Cox and S. Zucker, Inventiones Mathematicae 53, 1 (1979).

[26] For $n=9,10,11$ additional blowups are required [27].

[27] D. R. Morrison and W. Taylor, Central Eur. J. Phys. 10, 1072 (2012).

[28] W. Lerche, D. Lüst, and A. N. Schellekens, Nucl. Phys. B287, 477 (1987).

[29] We thank W. Lerche for discussions on this point.

[30] P. Griffiths and J. Harris, Principles of Algebraic Geometry (Wiley, New York, 1978).

[31] This is to be contrasted with the 6d SCFT sectors on the base $\mathbb{P}_{h}^{1}$ of $\mathbb{F}_{n}$ which do induce a worldsheet current on the heterotic string.

[32] N. Elkies, Banff Workshop 18w5190: Geometry and Physics of F-Theory (2018), https://www.birs.ca/events/2018/5-dayworkshops/18w5190.

[33] F. Baume, E. Palti, and S. Schwieger, J. High Energy Phys. 06 (2015) 039.

[34] C. Lawrie, S. Schäfer-Nameki, and J. M. Wong, J. High Energy Phys. 09 (2015) 144.

[35] M. Cvetič, D. Klevers, H. Piragua, and W. Taylor, J. High Energy Phys. 11 (2015) 204.

[36] N. Raghuram and W. Taylor, J. High Energy Phys. 10 (2018) 182. 